\title{
ALGORITHM DEFINING THE HYDRAULIC RESISTANCE COEFFICIENT BY LINES METHOD IN GAS-LIFT PROCESS
}

\author{
N.S. HAJIYEVA, N.A. SAFAROVA, AND N.A. ISMAILOV
}

Received 03 June, 2015

\begin{abstract}
A mathematical model of the process of gas-lift in the oil production is considered. In this process the motions of gas and gas-liquid mixture (GLM) in annular space and in the lift are described by the system of partial differential equations of hyperbolic type. Applying the straight line method the initial problem is reduced to the system of ordinary differential equations relatively to the volume of gas, gas-liquid mixture and their pressures. To determine the coefficient of hydraulic resistance $\lambda_{c}$ statistical data for the fixed well is used (volume of injected gas at the wellhead of the annular space and GLM at the end of lift) and on the basis of these results, constituting the corresponding functional, the functional gradient is derived that allows one to calculate $\lambda_{c}$. On the concrete example the statistical value of the coefficient of hydraulic resistance $\lambda_{\tilde{c}}$ and the new value of the coefficient of hydraulic resistance $\lambda_{c}$ are compared, it is shown that $\lambda_{c}$ differs from the real $\lambda_{\tilde{c}}$ on the order of $10^{-3}$.
\end{abstract}

2010 Mathematics Subject Classification: 49J20; 65K05; 65N40

Keywords: gas-lift, gas-liquid mixture, hydraulic resistance coefficient, gradient of the functional

\section{INTRODUCTION}

As it is known $[7,8,12,18,20]$, the gas-lift method for oil production is used when the fountain method impossible due to the lower reservoir pressure. The essence of the gas-lift method is that due to the energy of the injected gas, the liquid can be lifted to the ground surface. The motions $[2,18,19]$ of the injected gas and GLM are described by the partial differential equations of hyperbolic type. To solve the corresponding optimal control problems (feeding on the estuary such minimum gas volume, that the debit would be the highest) the method of lines are used, which leads the initial problem to the linear - quadratic optimal control problem for a system of ordinary differential equations $[1,3,6]$.

However, there are some significant parameters such as the coefficient of hydraulic resistance $\lambda_{c}$ in the lift, included in the equation of fluid motion in the lift (the identifications or the inversions problems $[16,17])$, which determines the generation rate of GLM. In this paper we focus on the choice the parameter $\lambda_{c}$, where choosing the corresponding quadratic functional, the posed problem is solved that allows one to 
calculate $\lambda_{c}$ [8]. According to the obtained formulas is suggested the algorithm and the program is made using Matlab. Thus, the offered algorithm allows to find the coefficient of hydraulic resistance $\lambda_{c}$ and the results coincide with accuracy $10^{-3}$ with data from mines.

\section{Problem Statement}

As it is known [1,3, 5, 7,9], the motions of the gas and GLM in the tubes are described by the system of partial differential equations of hyperbolic type:

$$
\left\{\begin{array}{l}
\frac{\partial P}{\partial t}=-\frac{c_{i}^{2}}{F_{i}} \frac{\partial Q}{\partial x}, \\
\frac{\partial Q}{\partial t}=-F_{i} \frac{\partial P}{\partial x}-2 a_{i} Q,
\end{array} \quad i=1,2,\right.
$$

where $P=P(x, t)$ - is pressure, $Q-$ is volume of gas and GLM, correspondingly, $c_{i}$ is the sound velocity in the gas and GLM, correspondingly, $2 a_{i}=\frac{g}{\omega_{c}^{i}}+\frac{\lambda_{c}^{i} \omega_{c}^{i}}{2 D_{i}}(i=$ $1,2) ; g, \lambda_{c}^{i},(i=1,2)-$ acceleration of gravity and hydraulic resistance in the gas and GLM, correspondingly, $\omega_{c}^{i},(i=1,2)$ - averaged over the cross section of the velocity of the gas and mixture in the annular zone and lift, correspondingly. $D_{i}, i=1,2$ are effective internal diameters of lift and annular space, $F_{i}, i=1,2-$ cross-sectional area of pump-compressor pipe and is also a constant on axes. Applying the straight line method and denoting $l=1 / 2 n$, we obtain [14] from (2.1)

$$
\left\{\begin{array}{l}
\frac{d P_{k}}{d t}=-\frac{c_{i}^{2}}{F_{i} l}\left(Q_{k}-Q_{k-1}\right), \\
\frac{d Q_{k}}{d t}=-\frac{F_{i}}{l}\left(P_{k}-P_{k-1}\right)-2 a_{i} Q_{k},
\end{array}\right.
$$

where

$$
F_{i}=\left\{\begin{array}{l}
F_{1}, 0<k \leq n, \\
F_{2}, n<k \leq 2 n,
\end{array} \quad c_{i}=\left\{\begin{array}{l}
c_{1}, 0<k \leq n, \\
c_{2}, n<k \leq 2 n .
\end{array}\right.\right.
$$

Note that for $k=n+1$ an equation (2.2) has the following form [10]

$$
\left\{\begin{array}{l}
\frac{d P_{n+1}}{d t}=-\frac{c_{2}^{2}}{F_{2} l} Q_{n+1}+\frac{c_{2}^{2}}{F_{2} l} Q_{n}+\frac{c_{2}^{2}}{F_{2} l} Q_{p l}, \\
\frac{d Q_{n+1}}{d t}=-\frac{F_{2}}{l} P_{n+1}+\frac{F_{2}}{l} P_{n}-2 a_{2} Q_{n+1}+\frac{F_{2}}{l} P_{p l},
\end{array}\right.
$$

where $Q_{p l}, P_{p l}$ - are volume flow and pressure of the reservoir at the bottom of the well.

After some transformations the system (2.2) can be reduced to

$$
\dot{x}=A\left(\lambda_{c}\right) x+B u+V,
$$

with initial condition

$$
X_{0}=\left[P_{1}^{0}, Q_{1}^{0}, \ldots, P_{2 n}^{0}, Q_{2 n}^{0}\right]^{\prime},
$$

where $X_{0}$ is the initial position of the well, $u$ is controlling influence and $A=\left[a_{i j}\right]$, 


$$
B=\left[b_{i j}\right], V=\left[v_{i j}\right] \text {, }
$$

$$
\begin{aligned}
& a_{i j}=\left\{\begin{array}{l}
a_{11}, \text { if } i=j \quad \text { and } 1 \leq i \leq n, \\
a_{12}, \text { if } i=j+1 \text { and } 1 \leq i<n-1, \\
a_{22}, \text { if } i=j \text { and } n<i<2 n \\
a_{21}, \text { if } i=j+1 \text { and } n-1<j \leq 2 n-1, \\
0, \text { in other cases, }
\end{array}\right. \\
& a_{11}=\left[\begin{array}{cc}
0 & -c_{1}^{2} / F_{1} l \\
-F_{1} / l & -2 a_{2}
\end{array}\right], a_{12}=\left[\begin{array}{cc}
0 & c_{1}^{2} / F_{1} l \\
F_{1} / l & 0
\end{array}\right], \\
& a_{21}=\left[\begin{array}{cc}
0 & c_{2}^{2} / F_{2} l \\
F_{2} / l & 0
\end{array}\right], a_{22}=\left[\begin{array}{cc}
0 & -c_{2}^{2} / F_{2} l \\
-F_{2} / l & -2 a_{2}
\end{array}\right], \\
& b_{i j}=\left\{\begin{array}{l}
c_{1}^{2} / F_{1} l, \quad \text { if } i=1, j=2, \\
F_{1} / l, \quad \text { if } i=2, j=1, \\
0, \quad \text { in other cases }
\end{array}\right. \\
& v_{i j}=\left\{\begin{array}{l}
c_{2}^{2} / F_{2} l, \quad \text { if } i=n+1, j=2, \\
F_{2} / l, \quad \text { if } i=n+2, j=1, \\
0, \quad \text { in other cases. }
\end{array}\right. \\
& x=\left[P_{1}, Q_{1}, P_{2}, Q_{2}, \ldots, P_{n}, Q_{n}, P_{n+1}, Q_{n+1}, \ldots, P_{2 n}, Q_{2 n}\right]^{\prime}
\end{aligned}
$$

Let's have some statistics, which at the given initial volumes of gas the debit $\tilde{Q}_{2 n}^{i}$ is measured at the output, i.e. $\tilde{Q}_{0}^{i}$, and $\tilde{Q}_{2 n}^{i}$ are known $(i=\overline{1, N})$, where $N$ is the number of measurements $[4,8]$.

It is required to find such values of the coefficient of hydraulic resistance $\lambda_{c}$ $[4,8,11,13]$, which system (2.3) will describe the motion of GLM in the lift, closer to practice (adequate mathematical model ). To solve this problem, the objective function based on the lowest square deviation from the real initial data $\tilde{Q}_{2 n}^{i}$ is constructed, i.e. it is required to minimize the functional

$$
f\left(a_{2}\left(\lambda_{c}\right)\right)=\sum_{i=1}^{N}\left[\tilde{Q}_{2 n}^{i}-Q_{2 n}^{i}\right]^{2} .
$$

Thus, the problem of defining the coefficient of hydraulic resistance $\lambda_{c}$ is reduced to the finding the gradient of functional (2.5). 


\section{CAlCulating the GRAdient OF The Functional (2.5)}

The general solution of equation (2.2) can be represented as follows

$$
\begin{aligned}
& x(t)=e^{A t} x_{0}+\int_{0}^{t} e^{A(t-\sigma)} B u d \sigma+\int_{0}^{t} e^{A(t-\sigma)} V d \sigma= \\
& =e^{A t} x_{0}+A^{-1}\left(e^{A t}-E\right) B u+A^{-1}\left(e^{A t}-E\right) V,
\end{aligned}
$$

where from the equation (3.1) $Q_{2 n}^{i}$ has the form

$$
Q_{2 n}^{i}=J \cdot x(T)
$$

where $J=[0,0,0,0, \ldots, 1], E-$ is the identity matrix.

In fact, $u$ is a function of the variable $t$. To get any value of the volume of gas or the corresponding value of the pressure, we can take them in the mouth of the well in the form of $\sin (\mathrm{bt}) \mathrm{u}_{0}$, that formula (3.1) turns into the following

$$
\begin{aligned}
& x(t)=e^{A t} x_{0}+\int_{0}^{t} e^{A(t-\sigma)} B u d \sigma+\int_{0}^{t} e^{A(t-\sigma)} V d \sigma=e^{A t} x_{0}+ \\
& +\int_{0}^{t} e^{A(t-\sigma)} B \sin (b \sigma) u_{0} d \sigma+\int_{0}^{t} e^{A(t-\sigma)} V d \sigma= \\
& =\left(e^{A t} x_{0}+E\left(e^{A t}-u_{0} \cos t-A \sin t\right) B u_{0}\right)\left(E-A^{2}\right)^{-1}+A^{-1}\left(e^{A t}-E\right) V .
\end{aligned}
$$

Then, substituting (3.1) and (3.2) in (2.5) we have

$$
\begin{aligned}
& f\left(a_{2}\left(\lambda_{c}\right)\right)=\sum_{i=1}^{N}\left[\tilde{Q}_{2 n}^{i}-J \cdot x(T)\right]^{2}= \\
& =\sum_{i=1}^{N}\left[\tilde{Q}_{2 n}^{i}-J e^{A} x_{0}^{i}-J A^{-1}\left(e^{A T}-E\right) B u-J A^{-1}\left(e^{A T}-E\right) V\right]^{2} .
\end{aligned}
$$

For the solution of the optimization problem (2.3) - (2.5) we find the gradient of the functional $f\left(a_{2}\left(\lambda_{c}\right)\right)$ and equating it to zero, we have 


$$
\begin{aligned}
& \frac{\partial f\left(a_{2}\left(\lambda_{c}\right)\right)}{\partial a_{2}\left(\lambda_{c}\right)}=2 \sum_{i=1}^{N}\left(\tilde{Q}_{2 n}^{i}-J e^{A T} x_{0}-\right. \\
& \left.-A^{-1}\left(e^{A T}-E\right) B u-J A^{-1}\left(e^{A T}-E\right) V\right) \times \\
& \times \sum_{i=1}^{N}\left(-J\left(\sum_{k=1}^{\infty} \sum_{j=1}^{k} \frac{T^{k}}{k !}\left(A\left(a_{2}\left(\lambda_{c}\right)\right)\right)^{j-1} \times\right.\right. \\
& \left.\times \frac{\partial A\left(a_{2}\left(\lambda_{c}\right)\right)}{\partial a_{2}\left(\lambda_{c}\right)}\left(A\left(a_{2}\left(\lambda_{c}\right)\right)\right)^{j-1}\right) x_{0}^{i}-J^{-1} \frac{\partial A\left(a_{2}\left(\lambda_{c}\right)\right)}{\partial a_{2}\left(\lambda_{c}\right)}{ }^{-1} B u- \\
& -\left(\sum_{k=1}^{\infty} \sum_{j=1}^{k} J \frac{T^{k}}{k !}\left(A\left(a_{2}\left(\lambda_{c}\right)\right)\right)^{j-1} \frac{\partial A\left(a_{2}\left(\lambda_{c}\right)\right)}{\partial a_{2}\left(\lambda_{c}\right)} \times\right. \\
& \left.\times\left(A\left(a_{2}\left(\lambda_{c}\right)\right)\right)^{j-1}\right) B u+J^{-1} \frac{\partial A\left(a_{2}\left(\lambda_{c}\right)\right)}{\partial\left(a_{2}\left(\lambda_{c}\right)\right)}{ }^{-1} V+ \\
& +J\left(--1 \frac{\partial A\left(a_{2}\left(\lambda_{c}\right)\right)}{\partial a_{2}\left(\lambda_{c}\right)}+1 \sum_{k=1}^{\infty} \sum_{j=1}^{k} \frac{T}{(k+1) !}^{k+1} \times\right. \\
& \left.\left.\times\left(A\left(a_{2}\left(\lambda_{c}\right)\right)\right)^{j-1} \frac{\partial A\left(a_{2}\left(\lambda_{c}\right)\right)}{\partial a_{2}\left(\lambda_{c}\right)}\left(A\left(a_{2}\left(\lambda_{c}\right)\right)\right)^{k-j}\right) V\right)=0 .
\end{aligned}
$$

Using the method [4] for the solution of the nonlinear equation we define $\lambda_{c}$ in the form

$$
\lambda_{c}=4 a_{2} D / \omega_{c}+2 D g / \omega_{c}^{2} .
$$

Thus, we formulate the following algorithm for finding $\lambda_{c}$.

\section{Algorithm:}

1. The initial data and parameters $A, B, V, u$ are introduced from (2.3);

2. The statistical (observations) data

$$
x_{0}=\left[P_{1}^{0 i}, Q_{1}^{0 i}, P_{2}^{0 i}, Q_{2}^{0 i}, \cdots, P_{n}^{0 i}, Q_{n}^{0 i}, P_{n+1}^{0 i}, Q_{n+1}^{0 i}, \cdots, P_{2 n}^{0 i}, Q_{2 n}^{0 i}\right]^{\prime}
$$

and the debit $\tilde{Q}_{2 n}^{i}$ from the practice for the same well $(i=\overline{1, N}, N$-is the number of measurements) are introduced;

3. The matrix $A, B$ are founded from (3.1)

$$
x(t)=\left[P_{1}, Q_{1}, \ldots, P_{n+1}, Q_{n+1}, P_{2 n}, Q_{2 n}\right] ;
$$

4. The functional $f\left(a_{2}\left(\lambda_{c}\right)\right)$ is formulated from (3.3);

5. The solution of equation (3.4) is founded using the steepest descent method and univariate method (golden section search method) [15].

6. The condition $\left|\frac{\partial f\left(a_{2}\left(\lambda_{c}\right)\right)}{\partial a_{2}\left(\lambda_{c}\right)}\right|<\varepsilon$ is checked out for sufficiently small number $\varepsilon$. If this condition is not satisfied we go to the step 2, else calculating procedure ends.

Example. Let's consider the realization of the proposed algorithm on the example [10]. After applying of the proposed algorithm we obtain that $\lambda_{c}=0.2357157$. Here $\tilde{\lambda}_{c}=0.23$, where $\tilde{\lambda}_{c}$ is the hydraulic resistance value from practice $[4,8]$. Note that 
$\lambda_{c}$ differs from $\tilde{\lambda}_{c}$ to the order $10^{-3}$, and it shows the efficiency of the proposed algorithm.

\section{CONCLUSiON}

The quadratic functional is formed for the solution of the indicated problem and it allows to find the coefficient of hydraulic resistance. The offered algorithm confirm the adequacy of the constructed mathematical model with practice.

\section{REFERENCES}

[1] A. P. Afanas'ev, S. M. Dzyuba, I. . Emelyanova, and A. Ramazanov, "Optimal control with feedback of some class of nonlinear systems via quadratic criteria," Appl. Comput. Math., vol. 15, no. 1 , pp. $78-87,2016$.

[2] F. A. Aliev, M. K. Ilyasov, and M. A. Jamalbekov, "Modeling of work of gas lift well," Reports of ANAS, vol. 22, no. 4, pp. 107-116, 2008, in Russian.

[3] F. A. Aliev, M. K. Ilyasov, and N. B. Nuriev, "Problems of modeling and optimal stabilization of the gas lift process," International Applied Mechanics, vol. 46, no. 6, pp. 709-717, 2010.

[4] F. A. Aliev and N. A. Ismailov, "Inverse problem to determine the hydraulic resistance coeffficient in the gaslift process," Appl. Comput. Math., vol. 12, no. 3, pp. 306-313, 2013.

[5] F. A. Aliev, N. A. Ismailov, H. Haciyev, and M. F. Guliyev, "A method to determine the coefficient of hydraulic resistance in different areas of pump-compressor pipes," TWMS J. Pure Appl. Math, vol. 7, no. 2, pp. 211-217, 2016.

[6] F. A. Aliev, N. A. Ismailov, E. V. Mamedova, and N. S. Mukhtarova, "Computational algorithm for solving problem of optimal boundary-control with nonseparated boundary conditions," J. Comput. Syst. Sci.Int., vol. 55, no. 2, pp. 700-711, 2016, doi: 10.1134/S1064230716030035.

[7] F. A. Aliev, N. A. Ismailov, and N. S. Mukhtarova, "Algorithm to determine the optimal solution of a boundary control problem," Automation and Remote Control, vol. 76, no. 4, pp. 627-633, 2015, doi: 10.1134/S0005117915040074.

[8] F. A. Aliev, N. A. Ismailov, and A. A. namazov, "Asymptotic method for finding the coefficient of hydraulic resistance in lifting of fluid on tubing," Journal of Inverse and Ill-posed Problems, vol. 23, no. 5, pp. 511-518, 2015.

[9] F. A. Aliev, N. A. Ismailov, A. A. Namazov, and M. F. Rajabov, "Algorithm for calculating the parameters of formation of gas-liquid mixture in the shoe of gas lift," Appl. Comput. Math., vol. 15, no. 3, pp. 370-376, 2016.

[10] F. A. Aliev, M. M. Mutallimov, I. M. Askerov, and I. S. Raguimov, "Asymptotic method of solution for a problem of construction of optimal gas-lift process modes Mathematical Problems in Engineering, vol. 2010, article id 191053, 11 pages," Mathematical Problems in Engineering, vol. 2010, 2010, article ID 191053, doi: 10.1155/2010/191053.

[11] A. D. Altshul, Hydraulic resistance. Moscow: Nedra, 1970, in Russian.

[12] R. L. Barashkin, "Modeling of gas liquid mixture motion in tubing of gas lift well," Automation, telemechanization and connection in the oil industry, vol. 5, pp. 41-46, 2011, in Russian.

[13] X. H., Y. H. Wang, H. Wang, and S. N. Balakrishnan, "Variable time impulse system optimization with continuous control and impulse control," Asian J. Control, vol. 16, no. 1, pp. 107-116, 2014, doi: 10.1002/asjc.769.

[14] K. K. Hasanov and T. S. Tanriverdiyev, "Optimal control problem describing by the cauchy problem for the first order linear hyperbolic system with two independent variables," TWMS J. Pure Appl. Math, vol. 6, no. 1, pp. 100-110, 2015. 
[15] D. M. Himmebblau, Applied nonlinear programming. New-York: Craw-Hill Book Company, 1972.

[16] N. Huang and C. F. Ma, "Two inversion-free iterative algorithms for computing the maximal positive definite solution of the nonlinear matrix equation," Appl. Comput. Math, vol. 14, no. 2, pp. 158-167, 2015.

[17] M. A. Mabrok, M. A. Haggag, and I. R. Petersen, "System identification algorithm for negative imaginary systems," Appl. Comput. Math, vol. 14, no. 3, pp. 336-348, 2015.

[18] A. K. Mirzadjanzadeh, I. M. Akhmetov, A. M. Khasaev, and V. I. Gusev, Technology and technique of oil production. Moscow: Nedra, 1986, in Russian.

[19] D. V. Shternlikht, Hydraulics. Moscow: Kolos, 2005, in Russian.

[20] V. I. Shurov, Technology and technique of oil production. Moscow: Nedra, 1983, in Russian.

Authors' addresses

N.S. Hajiyeva

Institute of Applied Mathematics, BSU, Z. Khalilov str.23, AZ1148 Baku, Azerbaijan

E-mail address: nazile.m@mail.ru

N.A. Safarova

Institute of Applied Mathematics, BSU, Z. Khalilov str.23, AZ1148 Baku, Azerbaijan

E-mail address: narchis2003@yahoo.com

N.A. Ismailov

Institute of Applied Mathematics, BSU, Z. Khalilov str.23, AZ1148 Baku, Azerbaijan

Institute of Information Technology of ANAS, B.Vakhabzade str.9, AZ1141 Baku, Azerbaijan

E-mail address: inao212@rambler.ru 